\title{
Correction to: A seasonal geometric INAR process based on negative binomial thinning operator
}

\author{
Shengqi $\operatorname{Tian}^{1} \cdot$ Dehui Wang $^{1} \cdot$ Shuai Cui $^{1}$
}

Published online: 12 March 2019

๑) Springer-Verlag GmbH Germany, part of Springer Nature 2019

\section{Correction to: Statistical Papers https://doi.org/10.1007/s00362-018-1060-7}

In the original publication of the article, the article title was incorrectly published as "A seasonal geometric INAR(1) process based on negative binomial thinning operator". However, the correct article title is "A seasonal geometric INAR process based on negative binomial thinning operator".

The original paper has been corrected.

Publisher's Note Springer Nature remains neutral with regard to jurisdictional claims in published maps and institutional affiliations.

The original article can be found online at https://doi.org/10.1007/s00362-018-1060-7.

Dehui Wang

wangdh@jlu.edu.cn

Shengqi Tian

tiansq15@mails.jlu.edu.cn

Shuai Cui

cuishuai@jlu.edu.cn

1 School of Mathematics, Jilin University, Changchun 130012, China 